\begin{tabular}{|c|l|}
\hline Title & Fiber-Based Bidirectional Solid-State Single Photon Emitter Based on Semiconductor Quantum Dot \\
\hline Author(s) & Sasakura, Hirotaka; Liu, X iangming; Odashima, Satoru; Kumano, Hidekazu; Muto, Shunichi; Suemune, Ikuo \\
\hline Citation & $\begin{array}{l}\text { A pplied Physics Express, 6(6), 065203 } \\
\text { https://doi.org/10.7567/APEX.6.065203 }\end{array}$ \\
\hline Issue Date & 2013 \\
\hline Doc URL & http://hdl.handle.net/2115/52863 \\
\hline Rights & ○ 2013 The Japan Society of A pplied Physics \\
\hline Type & article (author version) \\
\hline File Information & AP130456.pdf \\
\hline
\end{tabular}

Instructions for use 


\title{
Fiber-Based Bidirectional Solid-State Single-Photon Emitter Based on Semiconductor Quantum Dot
}

\author{
Hirotaka Sasakura*1, Xiangming $\mathrm{Liu}^{2}$, Satoru Odashima ${ }^{2}$, Hidekazu Kumano ${ }^{2}$, Shunichi Muto ${ }^{3}$, \\ and Ikuo Suemune ${ }^{2}$ \\ ${ }^{1}$ Creative Research Institution, Hokkaido University, Sapporo 001-0021, Japan \\ ${ }^{2}$ Research Institute for Electronic Science, Hokkaido University, Sapporo 001-0021, Japan \\ ${ }^{3}$ Department of Applied Physics, Hokkaido University, Sapporo 060-8628, Japan
}

\begin{abstract}
Fiber-based bidirectional photon detection from nanoscale emitters and photon antibunching behavior between two outputs of two single-mode fibers (SMFs) are experimentally demonstrated. Flakes containing the epitaxially grown quantum dots (QDs) are mechanically fixed by both sides with the edge faces of the SMF patch cables. The emitting photons from a single QD are directly taken out of both sides through the SMFs. Single-photon emission between two SMF outputs is confirmed by detecting nonclassical antibunching in second-order photon correlation measurements. This simple optomechanical alignment-free single-photon emitter is advantageous because of its robust stability of more than three months and low-cost fabrication.
\end{abstract}

Semiconductor quantum dots (QDs) are attractive nanoscale structures for solid-state nonclassical light sources and are expected to play key roles in a quantum information network. $^{1-3)}$ Above all, single QDs fabricated by epitaxial growth can serve as stable and bright photon emitters. Recently, significant progress of far-field optical coupling to a single semiconductor QD has been achieved by the Purcell effects with elaborated microcavity structures. ${ }^{4-9)}$ However, direct coupling of emitted photons to single-mode fiber (SMF) without exposure to the free-space, air or vacuum, is preferable for future applications. Indeed, various efforts have been made to directly couple a nanoscale photon emitter with a conventional SMF from the perspective of consistency with an existing optical fiber infrastructure. Several groups reported the efficient extraction of photons from a nanoscale emitter directly into an optical fiber by using a tapered fiber, ${ }^{10)}$ nanophotonic directional coupler, ${ }^{11)}$ integration of telluride fiber, ${ }^{12}$ and direct placing on the fiber facet by an atomic force microscope. ${ }^{13)}$ In these approaches, no optical loss occurred at coupling from the free-space into optical fibers. From a scientific as well as engineering viewpoint, the long-term stability is another of the most important conditions for a nanoscale photon emitter.

This study reports a simple fabrication of a bidirectional solid-state single-photon emitter based on epitaxially grown InAs QDs, which has a long-term stability and also a high po-

*E-mail address: hirotaka@cris.hokudai.ac.jp 
tentiality for a direct coupling to SMFs. The photons emitted from the single InAs QDs as single-photon emitters are taken out of both sides through the SMFs. Flakes of the epitaxial layers containing the InAs QDs are obtained by simply scratching the surface with an ordinary diamond cutter and are mechanically fixed by both sides with the edge faces of the SMF patch cables. Clear antibunching between the two outputs of two SMF patch cables is observed in second-order photon correlation measurements with superconducting single-photon detectors (SSPDs) and a time amplitude converter (TAC). Placing the solid-state single-photon sources and the SMFs in direct contact is useful from a practical perspective [Fig. 1(a)] because it offers robust stability and a solid-state optical as well as mechanical alignment. Moreover, this simple structure facilitates the low-cost fabrication of photon emitters and the sufficient stability of the output photon number from a scientific viewpoint.

The InAs QDs were grown to a density of $7 \times 10^{9} \mathrm{~cm}^{-2}$ on a GaAs buffer layer on a semi-insulating GaAs (001) substrate by molecular-beam epitaxy (RIBER, MBE32P). The low-temperature indium flush method was used to restrict the QD height to $2.8 \mathrm{~nm}$. The detailed growth conditions and overall optical characteristics are presented in ref. 14. To detect the emitted photons in both directions, we used flakes of the epitaxial layers. The flakes having a diameter and thickness of 1-20 $\mu \mathrm{m}$ were obtained by simply scratching the surface with an ordinary diamond cutter. These flakes were directly attached to the edge face of an FC/PC SMF patch cable (Corning, SMF-28) with a $\phi 900 \mu \mathrm{m}$ jacket by electrostatic forces. To mechanically fix the flakes and effectively extract the generated photons into two SMFs, the edge face of another SMF patch cable was directly connected to the opposite side of the flakes using an FC/PC-FC/PC joint (Thorlabs, ADAFC2). The FC/PC-FC/PC joint part with its embedded flakes was set in a liquid ${ }^{4} \mathrm{He}$ reservoir at $4.2 \mathrm{~K}$ [Fig. 1(b)].

We used a fiber-pigtailed laser diode (Thorlabs, LPS-830-FC) that emits at $830 \mathrm{~nm}$ as an excitation source with the laser beam arriving at the flakes from the right side of the SMF. To clean up laser spectral noise, a long-pass filter and two short-pass filters were inserted into the laser beam (Thorlabs, FEL0800 and FES0850, respectively). The QD emissions were extracted using two SMFs, corresponding to the reflection (right side) and transmission (left side) configurations in the free-space optical measurement system. To spatially separate the emissions in the reflection direction, a fiber-based dichroic beamsplitter module (Optoquest, custom-made product) was used. The emission was dispersed by a double-grating spectrometer (Acton, Spectrapro 2500i, $f=1.0 \mathrm{~m}$ ) and detected with a liquid-nitrogen-cooled InGaAs photodiode array (Roper, OMA-V1024). The typical exposure time was $5 \mathrm{~s}$ to obtain a photoluminescence $(\mathrm{PL})$ spectrum with a high signal-to-noise ratio.

The sharp peaks originating from single InAs QDs were observed at the reflection and transmission configurations under the excitation power density of $5.6 \mathrm{~W} / \mathrm{cm}^{2}$ [Fig. 1(b)]. At low-excitation power, the emission $X$ is centered at $1071.12 \mathrm{~nm}$ with a $76 \mu \mathrm{eV}$ full width at 
half maximum (hereinafter called the $X$ line), which corresponds to the ground-state emission in the ensemble PL spectra. $\left.{ }^{14}\right)$ The observed intensities of the $X$ line in each configuration were almost identical, signifying that the present system that uses flakes sandwiched between SMF patch cables naturally forms a spatial Hanbury Brown and Twiss (HBT) interferometer without an additional bifurcation. Note that non-degraded PL spectra are also observed after three months with ten thermal cycles from $4.2 \mathrm{~K}$ to $300 \mathrm{~K}$.

Figures 2(a) and 2(b) show the excitation power dependence of the single InAs QD PL spectrum and detected photon count rate of the $X$ line selected with a 0.5 -nm-wide band-pass filter (Optoquest: custom-made product) in the transmission configuration. At low-excitation power, the $X$ line dominates, implying that the QD emitting the $X$ line optically couples well with the SMF core. With increasing excitation power, additional PL lines appear, which are exciton complexes of the QD and/or emissions originating from other QDs, and the photon count rate of the $X$ line linearly increases and saturates at $\sim 10 \mathrm{~W} / \mathrm{cm}^{2}$. At the highest excitation power density $\left(21.1 \mathrm{~W} / \mathrm{cm}^{2}\right)$, background photons appear, suggesting that a large number of QDs weakly couple with the SMFs. We note that the present system corresponds to the standard micro-PL measurements in the far field using a collective lens with a low numerical aperture $\leq 0.14$.

For the autocorrelation measurements, the output of each SMF is filtered with the 0.5-nmwide band-pass filter to select the $X$ line and three long-pass filters (Asahi Spectra, LIX900 and Thorlabs, EFL1000) to suppress background photons, i.e., reflection and transmission of the excitation laser at the interface of both SMF patch cables and other unwanted emissions originating from other QDs. The optical transmission loss of each part in selecting the $X$ line was $\sim 5 \mathrm{~dB}$. The $X$ line was then sent to an SSPD (Single Quantum, custom-made product). The total length of SMF from the QD to the SSPD was $\sim 50 \mathrm{~m}$, which indicates that the attenuation is negligible $(\sim 0.01 \mathrm{~dB})$. The detection efficiency and dark count of each SSPD were $\sim 15 \%$ and $\sim 50 \mathrm{cps}$, respectively. ${ }^{15)}$ The autocorrelation measurements of the $X$ line were performed between the right and left ports with an optical delay of $\sim 100$ ns to compensate for the internal electronic delay in the SSPD driver and in the TAC board (Becker \& Hickl, SPC-130). The single count rate of the $X$ line at each SSPD was $\sim 8 \mathrm{kHz}$ with an excitation power of $5.6 \mathrm{~W} / \mathrm{cm}^{2}$. A histogram of the normalized coincidence counts with time bins of 244 ps and an integration time of $10.5 \mathrm{~h}$ is shown in Fig. 2(c). The data exhibits the well-known antibunching dip at a zero time delay and is fit with the second-order correlation function $g_{b}^{(2)}(\tau)=1-\left[1-g^{(2)}(0)\right] \rho^{2} \exp (-|\tau| / t)$, where $\rho[=S /(S+B)]=0.99$ related to the signal-tonoise ratio ${ }^{16)}$ and $t\left[=t_{l} t_{p} /\left(t_{l}+t_{p}\right)\right]=0.54 \pm 0.10 \mathrm{~ns}$, where $t_{l}$ is the lifetime of the $X$ line and $t_{p}$ is the inverse pumping rate. At zero delay, the function gives $g^{(2)}(0)=0.26 \pm 0.05$, suggesting that the single InAs QD emits nonclassical light. Figures 2(d) and 2(e) show a normalized coincidence under excitation powers of 2.82 and $1.27 \mathrm{~W} / \mathrm{cm}^{2}$, respectively. Upon decreasing 
the excitation power, which corresponds to increasing $t_{p}$, the full width at half maximum of the antibunching dip gradually spreads up to $\sim 2.24 \mathrm{~ns}$, suggesting that $t$ is limited by $t_{p}$ under our measurement conditions. Although narrow antibunching appears in Figs. 2(c)2(e), a broad bunching extending over \pm 10 ns near the narrow antibunching dip appears for an excitation power of $5.6 \mathrm{~W} / \mathrm{cm}^{2}$. These bunching photon statistics are suppressed under low-power excitation, which we attribute to an enhanced probability of the exciton state $(X)$ repumping after emitting a photon ${ }^{17)}$ or the spectral diffusion effects due to environmental fluctuations. ${ }^{18,19)}$ Note that the long-term stability of present device enables us to validate nonclassical light characteristics not only without opto-mechanical alignment, but also under pulsed optical excitation which generates single photons on demand. ${ }^{20)}$

In summary, we demonstrated single-photon emission from the flakes containing epitaxially grown InAs quantum dots. The emitting photons originating from the single InAs quantum dots were extracted bidirectionally through single-mode fibers directly attached to the flakes. The photon antibunching behavior was confirmed by second-order photon correlation measurements with the superconducting single-photon detectors and time amplitude converter. These experimental results suggest that a simple single-photon emitter based on a single quantum dot sandwiched by two single-mode fiber patch cables has significant potential for robust long-term stability and for the fabrication of solid-state optomechanical alignment and low-cost photon emitters. Moreover, a bidirectional photon detection approach has potential to send a photon pair generated by a nanoscale emitter to separate directions without optical loss in coupling from free space into an optical fiber.

\section{Acknowledgement}

This work was supported in part by the Sumitomo Foundation and Nikki-Saneyoshi Foundation. X.L., H.K., and I.S. acknowledge funding from SCOPE. 

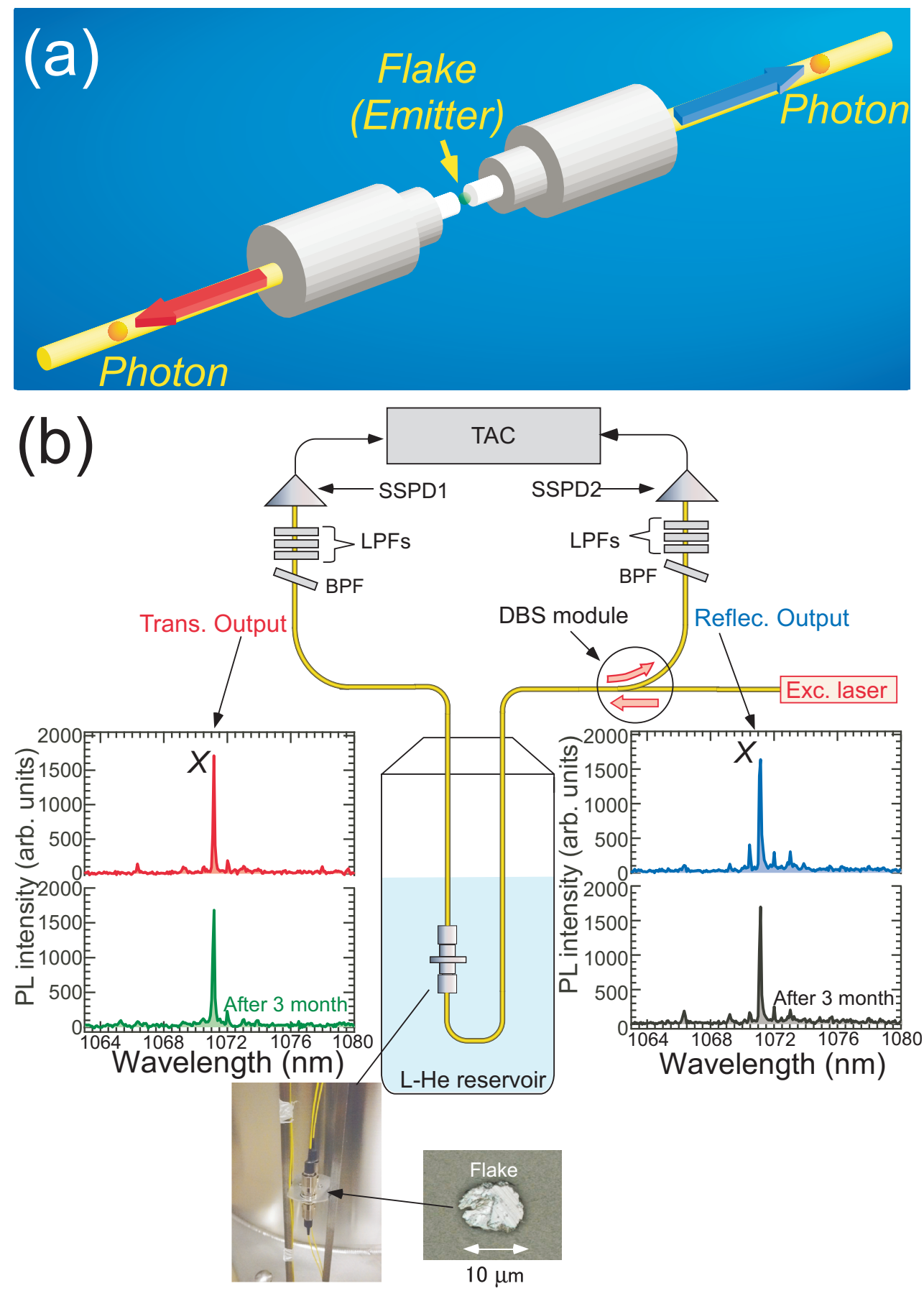

Fig. 1. Schematic of SMF-embedded solid-state single-photon emitter (a) and experimental setup of second-order photon correlation (b). (Inset): Measured PL spectra in transmission (Trans. Output) and reflection (Reflec. Output) configurations. The green and black PL spectra were remeasured after three months with ten thermal cycles. LPF is a long-pass filter, BPF is a 0.5-nm-wide band-pass filter, DBS is a dichroic beamsplitter, TAC is a time amplitude converter, and SSPD is a superconducting single-photon detector. 

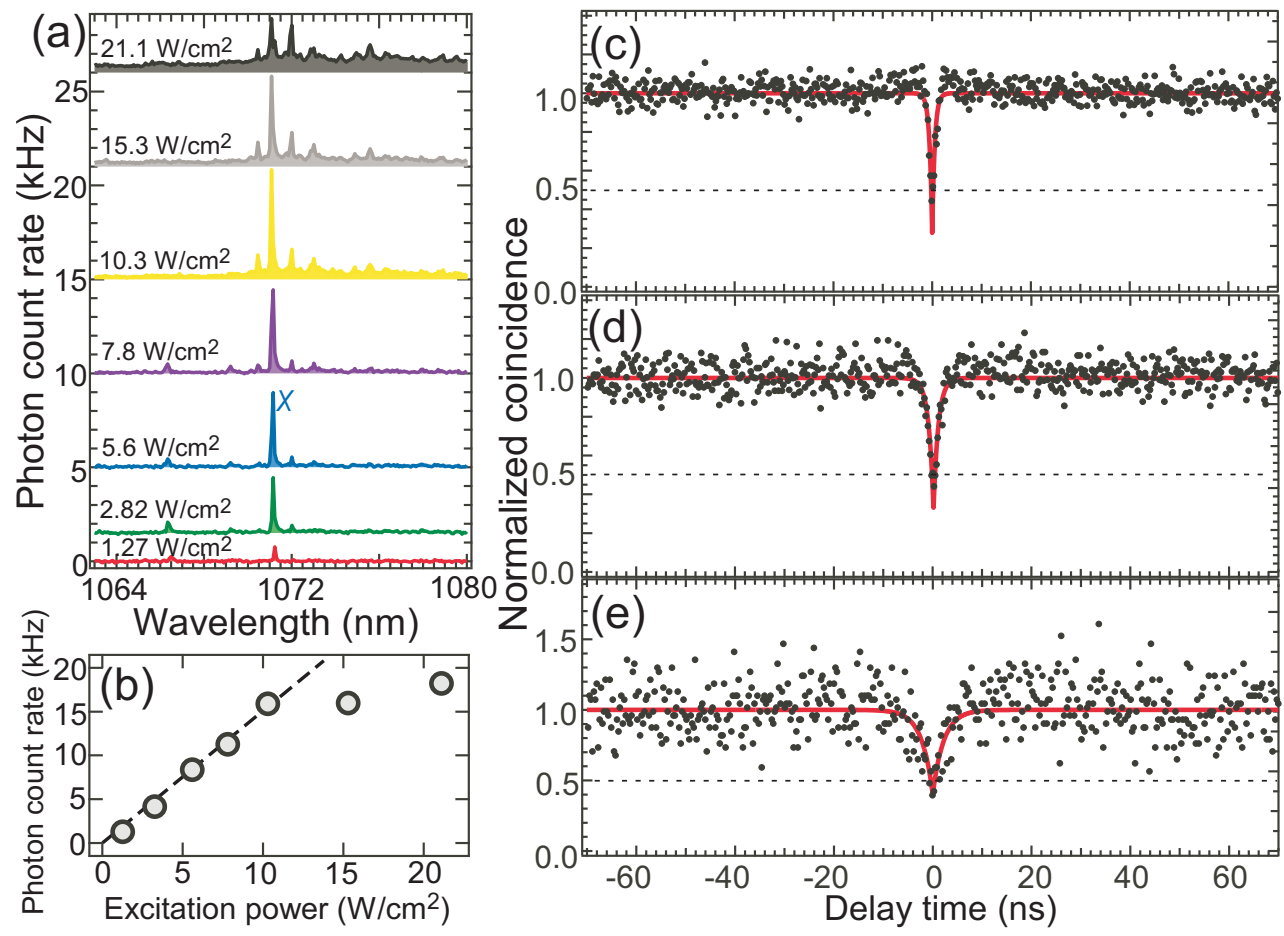

Fig. 2. Excitation power dependence of PL spectrum (a) and photon count rate of the wavelength range selected with the 0.5 -nm-wide band-pass filter (b) in the transmission configuration. The dashed line is a guide to the eye. (c)-(e) Second-order photon correlation is recorded using a TAC employing two SSPDs. Normalized histograms of the autocorrelation measurement of the $X$ line (solid circles) at excitation powers of 5.6 (c), 2.82 (d), and $1.27 \mathrm{~W} / \mathrm{cm}^{2}$ (e). Excitation wavelength is $830 \mathrm{~nm}$ and the time bins are $244 \mathrm{ps}$. The integration time and count rates are $10.5 \mathrm{~h}$ and $\sim 16 \mathrm{kHz}$ (c), $7.5 \mathrm{~h}$ and $\sim 8$ $\mathrm{kHz}$ (d), and $14.5 \mathrm{~h}$ and $\sim 3 \mathrm{kHz}$ (e), respectively. The solid (red) lines are fits, indicating a dip in the correlation function of $g^{(2)}(0)=0.26$ and $t=0.54 \mathrm{~ns}(\mathrm{c}), 0.32$ and $1.0 \mathrm{~ns}(\mathrm{~d})$, and 0.34 and $2.24 \mathrm{~ns}(\mathrm{e})$. 


\section{References}

1) D. D. Awschalom, N. Samarth, and D. Loss: Semiconductor Spintronics and Quantum Computation (Springer, Berlin, 2002).

2) A. J. Shields: Nat. Photonics 1 (2007) 215.

3) Jeremy L. O’Brien, Akira Furusawa, and Jelena Vǔcković: Nat. Photonics 3 (2009) 687.

4) E. Moreau, I. Robert, L. Manin, V. Thierry-Mieg, J. M. Gérard, and I. Abram: Physica E 13 (2002) 418.

5) C. Santori, D. Fattal, J. Vuckovic, G. S. Solomon, and Y. Yamamoto: New J. of Phys. 6 (2004) 89.

6) J. Claudon, J. Bleuse, N. S. Malik, M. Bazin, O. Jaffrennon, N. Gregersen, C. Sauvan, P. Lalanne, and J. -M. Gérard: Nat. Photonics 4 (2010) 174.

7) M. E. Reimer, G. Bulgarini, N. Akopian, M. Hocevar, M. B. Bavinck, M. A. Verheijen, E. P.A.M. Bakkers, L. P. Kouwenhoven, and V. Zwiller: Nat. Commun. 3 (2012) 737.

8) K. Takemoto, M. Takatsu, S. Hirose, N. Yokoyama, and Y. Sakuma: J. Appl. Phys. 101 (2007) 081720.

9) A. Dousse, J. Suffczyński, A. Berveratos, O. Krebs, A. Lemaître, L. Sagnes, J. Bloch, P. Voisin, and P. Senellart: Nature 466 (2010) 217.

10) M. Fujiwara, K. Toubaru, T. Noda, H.-Q. Zhao, and S. Takeuchi: Nano Lett. 11 (2011) 4362.

11) M. Davanco, M. T. Rakher, W. Wehsceider, D. Schuh, A. Badolato, and K. Srinivasan: Appl. Phys. Lett. 99 (2011) 121101.

12) M. R. Henderson, B. C. Gibson, H. Ebendorff-Heidepriem, K. Kuan, S. Afshar V., J. O. Orwa, I. Aharonovich, S. Tomljenovic-Hanic, A. D. Greentree, S. Prawer, and T. M. Monro: Adv. Mater. 23 (2011) 2806.

13) T. Schröder, A. W. Schell, G. Kewes, T. Aichele, and O. Benson: Nano Lett. 11 (2011) 198.

14) H. Sasakura, S. Kayamori, S. Adachi, and S. Muto: J. Appl. Phys. 102 (2007) 013515.

15) S. N. Dorenbos, P. Forn-Díaz, T. Fuse, A. H. Verbruggen, T. Zijlstra, T. M. Klapwijk, and V. Zwiller: Appl. Phys. Lett. 98 (2011) 251102.

16) C. Becher, A. Kiraz, P. Michler, A. Imamoglu, W. V. Schoenfeld, P. M. Petroff, L. Zhang, and E. Hu: Phys. Rev. B 63 (2001) 121312(R).

17) D. V. Regelman, U. Mizrahi, D. Gershoni, E. Ehrenfreund, W. V. Schoenfeld, and P. M. Petroff: Phys. Rev. Lett. 87 (2001) 257401. 
18) G. Sallen, A. Tribu, T. Aichele, R. André, L. Besombes, C. Bougerol, M. Richard, S. Tatarenko, K. Kheng, and J.-Ph. Poizat: Nat. Photonics 4 (2010) 696.

19) M. Abbarchi, T. Kuroda, T. Mano, M. Gurioli, and K. Sakoda: Phys. Rev. B 86 (2012) 115330.

20) K. Takemoto, Y. Nambu, T. Miyazawa, K. Wakui, S. Hirose, T. Usuki, M. Takatsu, N. Yokoyama, K. Yoshino, A. Tomita, S. Yorozu, Y. Sakuma, and Y. Arakawa: Appl. Phys. Express 3 (2010) 092802. 